\title{
Genetic Counselling in a Familial Deletion 18p Syndrome
}

\author{
Cozaru GC ${ }^{1}$, Așchie $\mathbf{M}^{1,2^{*}}$, Mitroi $A^{1}$, Poinăreanu $\mathbf{I}^{1,2}$ and Căpățînă $D^{1}$ \\ ${ }^{1}$ Department of Medicine, Sf. Apostol Andrei, Constanta County Emergency Clinical Hospital, Romania \\ ${ }^{2}$ Department of Medicine, Ovidius, University Constanta, Romania
}

*Corresponding author: Mariana Așchie, Department of Medicine, Sf. Apostol Andrei, Constanta County Emergency Clinical Hospital, Romania, Tel: +40745043505; Email: aschiemariana@yahoo.com

Received date: November 18, 2015; Accepted date: December 13, 2015; Published date: February 27, 2016

Copyright: ( 2016 Cozaru GC, et al. This is an open-access article distributed under the terms of the Creative Commons Attribution License, which permits unrestricted use, distribution, and reproduction in any medium, provided the original author and source are credited.

\begin{abstract}
Deletion of $18 p$ syndrome is characterized by dysmorphic features, growth deficiencies, and mental retardation with a poor verbal performance. Until now, few families have been described with limited clinical description. We report transmission of deletion 18p from a mother to his son. The proband is 8 years old and has short stature, dysmorphic features, polymorphous dyslalia and moderate mental retardation. The mother also presents dysmorphic features, mild mental retardation and has better verbal abilities than his son. Management needs to be handled by a multidisciplinary team and includes speech therapy, hormonal (if necessary) and psychological care. Genetic counselling for these patients should take into account these new data, especially in regard of a wider variability of intellectual outcomes and better verbal performance.
\end{abstract}

Keywords: Facial dysmorphism; Mental retardation; Familial syndrome; Chromosomal deletion

\section{Introduction}

Deletion of $18 \mathrm{p}$ syndrome (del18p), is a chromosomal aberration described by the french geneticist Jean de Grouchy in 1963, known to be compatible with long-term survival. About 1 in 50,000 babies is born with a deletion of $18 \mathrm{p}$ and more than one hundred cases have been reported. Most reports suggest that $18 \mathrm{p}$ deletions affect girls more often than boys (female to male ratio is $3: 2$ ). Phenotypic manifestations of this deletion are very sparse at birth, but during life may consist in: mental retardation, developmental delay and learning difficulties, growth retardation, craniofacial dysmorphism (round face, dysplastic ears, ptosis, epicanthal folds, hypertelorism, low nasal bridge, wide mouth and dental anomalies) and abnormalities of the limbs (clinodactyly of the fifth finger), genitalia, brain (for example holoprosencephaly, in $10 \%$ of the cases), eyes, and heart. It seems that beside the typical manifestations of that syndrome, the girl had an abnormality of thyroxine synthesis [1], most likely a coupling defect. After Jones KL (2006), there is a significant discrepancy between verbal and non-verbal performance, verbal performance being more severely affected.

First familial cases (mother and her son and daughter were affected) were described by Uchida et al. (1965) and Velagaleti et al. (1996) [2,3]. Most cases of deletion $18 \mathrm{p}$ cases have been sporadic, are supposed to originate from de novo deletions, which accounts for $85 \%$ of cases, and in the majority of the individuals, the deletion is the only chromosome change present. New cytogenetic techniques have shown that in some cases, the deletion results from a more complicated chromosome rearrangement, like an unbalanced translocation that may lead to $18 \mathrm{p}$ and a duplication of another piece of chromosome. In this case, predicting what type of problems a child might have is more difficult. People with an unbalanced translocation may have features of $18 \mathrm{p}$ - as well as features of the chromosome duplication.

\section{Case presentation}

\section{Objective}

This paper aims to highlight the importance of preconception genetic counselling and prenatal diagnosis in case there is the risk of transmission of chromosomal anomalies. We consider that an informed decision is important for the following reasons: promotes the best interests, protects the pregnant woman against the risk of being misled or forced to decide in contradiction with personal moral or religious beliefs, respects the autonomy of the couple and, last but not least, protects the doctor from legal disputes.

\section{Participants}

We report a transmission of $18 \mathrm{p}$ chromosome deletion from a mother to his son who presented in our department for investigation and diagnosis. Parents want to know what are the characteristics of the evolution of the disease in their child, diagnosed with a genetic syndrome that associates various malformations or the risk of developing certain diseases along the life. Also, they requested genetic counselling and calculation of the risk of recurrence in offspring.

\section{Proband}

The proband is an 8 year-old boy with short stature, which was born at 37 weeks of gestation; birth weight was $2800 \mathrm{~g}$, length at birth $50 \mathrm{~cm}$. The child was brought to medical attention at the age of 1.5 years because behavioral problems and developmental delay. His evolution showed many respiratory infections, recurrent otitis media that caused moderate conductive hearing loss. On examination, we noted: weight is at the 50th centile, height at $3^{\text {rd }}$ centile and head circumference at 50th centile. Fenotype is marked by dysmorphic features: brachycephaly, broad neck with low posterior hairline, large and protruding ears, the face is rectangular, hypertelorism, ptosis, epicanthal folds, low nasal bridge, the mouth is large with a prominent philtrum and a high-arched palate, downturning corners of the mouth 
Page 2 of 4

(Figure 1). He has also: small hands and clinodactyly of the fifth fingers (which was observed in about $10 \%$ and $20 \%$ of the cases, respectively). Neurological examination diagnosed: spastic tetra-paresis, with a delay in the psycho-motor evolution. Cerebral imaging revealed: right fronto-parietal polimicrogirie, hipomielinization, bilateral frontoparietal lesions of the subcortical white matter (which are suggestive for vasculitis). Abdominal ultrasound was performed and revealed no abnormalities. Endocrine evaluation was also normal, including thyroid stimulating hormone and insulin-like growth factor 1 . Immune function was adequate with immunoglobulin A within normal range.

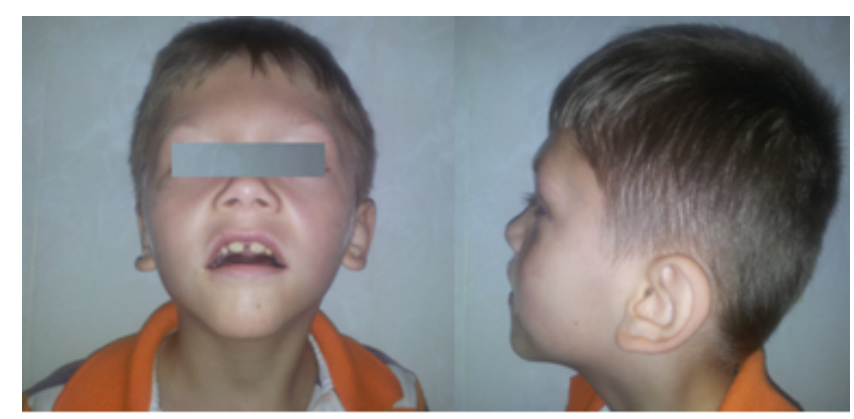

Figure 1: The proband: 8 years-old boy with dysmorphic features.

We have noticed some behavioral problems, which include: medium intellectual disability $(\mathrm{IQ}=45)$, relatively poor expressive communication and polymorphous dyslalia.

\section{Mother}

The mother is 40 years old, with a history of infectious and reproductive conditions. Her pubertal development is described as normal with menarche at age 12, and she leaves an active and independent life. Women with del (18p) are fertile and seem to have a normal miscarriage rate. In this case, the mother presented three miscarriages before the proband (between 6 and 8 weeks of gestation) and no analysis was performed on the fetus (including proband), then born a healthy girl (17 years old) and proband. Phenotypical observation revealed the following features: low posterior hairline, large protruding ears, rectangular face, epicanthal folds, large mouth with prominent philtrum, high-arched palate and down-turning corners of the mouth. She showed small hands and clinodactyly of her fifth fingers as well. Her height is $155 \mathrm{~cm}$, which is around the fifth percentile. Her cognitive performance is within the borderline to mild mental retardation range, she has better verbal abilities than his son and learning difficulties especially in mathematics.

\begin{tabular}{|l|l|l|}
\hline \multirow{2}{*}{ Characteristic features } & \multicolumn{2}{|l|}{ Family member } \\
\cline { 2 - 3 } & Proband (son) & Mother \\
\hline Age (years) & 8 & 32 \\
\hline $\begin{array}{l}\text { Physically developmental } \\
\text { delay }\end{array}$ & + & + \\
\hline Dysmorphic features & + & + \\
\hline Mental retardation (MR) & ++ & $+/-$ \\
\hline Short Stature & + & + \\
\hline
\end{tabular}

\begin{tabular}{|c|c|c|}
\hline Abnormalities of the limbs & + & + \\
\hline Brain abnormalities & + & - \\
\hline Chromosomal anomaly & $\operatorname{del}(18 p 11.2)$ & $\operatorname{del}(18 p 11.2)$ \\
\hline Observation & $\begin{array}{lr}\text { Poorer } & \text { verbal } \\
\text { performance } & \text { than } \\
\text { mother } & \end{array}$ & $\begin{array}{l}\text { Borderline to mild MR } \\
\text { Two miscarriages } \\
\text { Better verbal abilities than } \\
\text { his son }\end{array}$ \\
\hline
\end{tabular}

Table 1: Phenotypical characteristics.

\section{Genetic analyses}

Based on the clinical exam, we performed karyotype from a peripheral blood sample for proband and the mother. Chromosomal analysis of heparinized peripheral blood lymphocytes in sodium heparin was carried out using GTG banding in synchronized 72-hour cultures in complete RPMI 1640. The karyotype showed the same chromosomal deletion for two subjects: 46, XX, del (18) (p11.2) (Figures 2A and B). To confirm the diagnosis, we performed metaphasic fluorescent in situ hybridization analysis (FISH), according to the standard procedure, with $18 \mathrm{p} / 18 \mathrm{q}$ subtelomeric probes. The chromosomes were counterstained with DAPI and visualized on a Zeiss Axioscope microscope. On both cases, the results showed deletion on the short arm of one of the 18 chromosome (Figures $2 \mathrm{~A}$ and $\mathrm{B})$. The deletion appeared to encompass the almost whole short arm as evidenced by the GTG banding procedure of a 550-band level and in situ hybridization.

$$
\begin{aligned}
& 3 \text { (6 IL } 11 \text { il }
\end{aligned}
$$

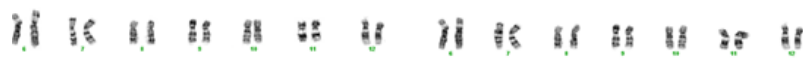

$$
\begin{aligned}
& \text { 4. }
\end{aligned}
$$

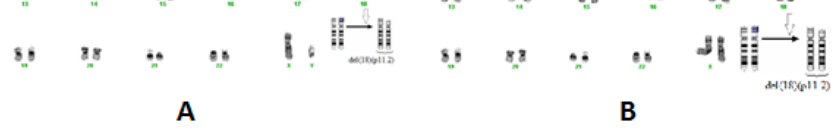

Figure 2: Giemsa-banding karyotype analysis revealed $18 \mathrm{p}$ deletion in the proband, respectively, on his mother - 46, XX, del(18)(p11.2).

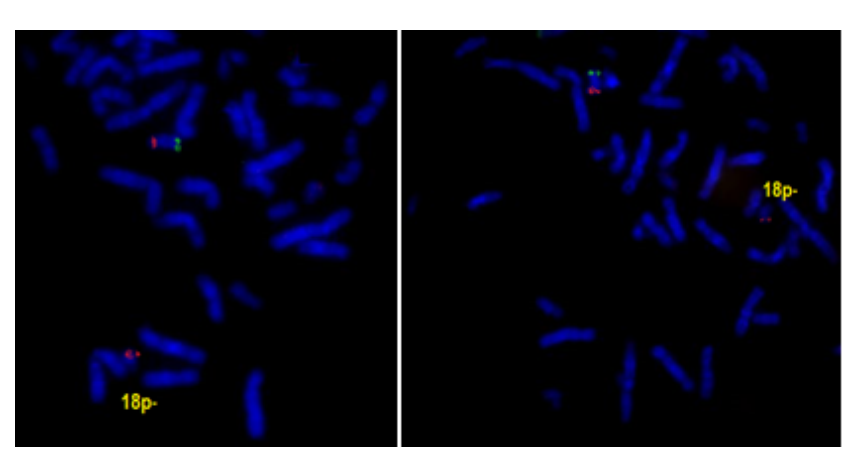

Figure 3: In situ hybridization 18p (green) and 18q (red) with subtelomeric probes showing $18 \mathrm{p}$ deletion in the proband, respectively his mother. 


\section{Discussion}

Birth of a child with chromosomal syndrome is a tragedy for any family. Physicians must pay attention to the psychological concerns of the family because identifying a genetic cause of the disease amplifies the psychological impact of a diagnosis. It is essential for parents to understand that the evolution and prognosis of the disease of their child are assessed according to the complexity of the chromosomal abnormalities. The importance of genetic diagnosis lies in the fact that it can explain the clinical features and evolution. Taking into account the fact that it is a familial case of transmission of chromosomal fault anomaly, it is essential to provide genetic counselling and correct risk calculation.

An ethical dilemma that the doctor has to bring to the attention of the pregnant woman/ the couple is that the prognosis may not be determined by prenatal diagnosis, the evolution of the disease ranging within some statistical limits. Although each anomaly has a characteristic clinical picture, common characteristics of unbalanced chromosomal anomalies consist in major multiple congenital anomalies and marked delay of uterine growth [4]. Related to different studies is very important to determine the exact size of the deletion for identifying critical regions and candidate genes, which may contribute to the features of $18 \mathrm{p}$ - syndrome. It is known that is a correlation between the breakpoints (critical region p11.1 - p11.21) and the degree of learning difficulties. It suggests that patients with a deletion distal to this point have normal intelligence or only very mild learning difficulties $[5,6]$. If the breakpoints involve the distal half of $18 p$ the child has post-natal growth retardation and seizures. Ptosis and short neck have been mapped to the proximal half of $18 p[6,7]$.

This report sheds new lights on the familial del (18p) syndrome. In our cases, the specific phenotype with dysmorphic clinical examination was essential in order to identify the cause of manifestations and to perform the proper genetic testing. The intellectual outcome is relatively constant throughout the same family. A similar case was described by the Uchida et al., with mother having $88 \%$ mosaic for del (18p) and an IQ of 75. Her child also had a developmental delay and mental retardation (IQ of 65\%). In 1996, Velagaleti et al., presented a familial case, having an 18p11.2 deletion, mother and her daughter with mild mental retardation. In our case, cognitive performance was more variable than previously suggested within the same family. The proband share moderate mental retardation while the mother has borderline to mild mental retardation. We noted that such variability has been observed also in other cases of familial deletions [8,9]. On different reported paper, del(18p) cases were associated with poorer verbal performance than non-verbal noted that slowness of motion and action was more of a problem to some study participants than mental retardation $[7,9,10]$. In the family we have investigated, language performance was more delayed than mental performance, and the mother of the proband has better verbal abilities than her son. Also, the case stands out by the presence of the neurologic lesions and delay in neuro-motor development, which severely impacts the prognosis. The complexity of the case imposes a multidisciplinary approach, by means of a complex team of specialists, in order to recuperate the neuro-motor delay, logopaedic and specific psychological counselling as well as recurrent check-ups for the reevaluation of the psycho-somatic development and improvement of intellective performances.

\section{Inheritance}

The possibility of having another pregnancy with $18 \mathrm{p}$ deletion depends on the parents' chromosomes. Normally, if both parents have normal karyotype, the deletion is very unlikely to happen again. In case one parent (like our case) has a chromosome rearrangement involving $18 \mathrm{p}$, the possibility is greatly increased by having other affected pregnancies. In the majority of cases showed mothers carrying babies with a del (8p) experienced no pregnancy problems, had a normal delivery and only discovered their baby was affected after the birth.

Until now, in all family cases with del (18p), were maternally transmitted an no paternal transmission has been described in our knowledge. Molecular characterization of $18 \mathrm{p}$ de novo deletions showed that women transmit unbalanced chromosome aberrations more frequently than men. Is still unknown specifically what are the cytogenetic background that could affect male fertility.

However, in our case if parents want to have another child, we recommend prenatal diagnosis (including chorionic villus sampling or amniocentesis, to test the baby's chromosomes) taking into account the chromosomal status of the mother and also the age over 35 years $[11,12]$. They have also the opportunity to reduce the risk of recurrence opting for prenatal and pre-implantation genetic diagnosis (PGD), that requires the use of in-vitro fertilization and embryo biopsy, and only healthy embryos are transferred to the mother's uterus $[13,14]$

\section{Conclusion}

Phenotypical features were quite similar between proband and his mother and in accordance with the usual phenotype of del (18p), but more severe at proband. Recognizing a genetic condition is vital because it may alter treatment outcome or therapy options. To avoid placing additional stress on the family, we should not delay investigation of abnormal development. Diagnosing a chromosomal abnormal condition also enables health care providers to both estimate the chance of recurrence in offsprings and discuss the availability of diagnostic testing for other family members.

\section{References}

1. Bühler EM (1983) Unmasking of heterozygosity by inherited balanced translocations. Implications for prenatal diagnosis and gene mapping. Ann Genet 26: 133-137.

2. Uchida IA, Mcrae KN, Ray M (1965) Familial Short Arm Deficiency of Chromosome 18 Concomitant with Arhinencephaly and Alopecia Congenita. Am J Hum Genet 17: 410-419.

3. Velagaleti GV, Harris S, Carpenter NJ, Coldwell J, Say B (1996) Familial deletion of chromosome 18 (p11.2). Ann Genet 39: 201-204.

4. Caba L, Panzaru M, Vicol MC, Braha EE, Popescu R, et al. (2012) Diagnostic prenatal invaziv versus noninvaziv: controverse si dileme etice. Revista Româna de Bioetica 10: 29-37.

5. Portnoï MF, Gruchy N, Marlin S, Finkel L, Denoyelle F, et al. (2007) Midline defects in deletion 18p syndrome: clinical and molecular characterization of three patients. Clin Dysmorphol 16: 247-252.

6. Wester U, Bondeson ML, Edeby C, Annerén G (2006) Clinical and molecular characterization of individuals with $18 \mathrm{p}$ deletion: a genotypephenotype correlation. Am J Med Genet A 140: 1164-1171.

7. Brenk CH, Prott EC, Trost D, Hoischen A, Walldorf C, et al. (2007) Towards mapping phenotypical traits in 18p- syndrome by array-based comparative genomic hybridisation and fluorescent in situ hybridisation. Eur J Hum Genet 15: 35-44. 
Citation: Cozarua GC, A?chie M, Mitroia A, Poinareanu I, Capa?înaa D (2016) Genetic Counselling in a Familial Deletion 18p Syndrome. Clinics Mother Child Health 13: 214. doi:10.4172/2090-7214.1000214

Page 4 of 4

8. Adeyinka A, Adams SA, Lorentz CP, Van Dyke DL, Jalal SM (2005) Subtelomere deletions and translocations are frequently familial. Am J Med Genet A 135: 28-35.

9. Jones KL (2006) Deletion $18 \mathrm{p}$ syndrome. In: Smith's recognizable patterns of human malformation (6th edn) Philadelphia, Elsevier Saunders, USA, pp. 60-61.

10. de Ravel TJ, Thiry P, Fryns JP (2005) Follow-up of adult males with chromosome 18p deletion. Eur J Med Genet 48: 189-193.

11. Baker E, Hinton L, Callen DF, Haan EA, Dobbie A, et al. (2002) A familia cryptic subtelomeric deletion $12 \mathrm{p}$ with variable phenotypic effect. Clin Genet 61: 198-201.
12. Barber JC (2005) Directly transmitted unbalanced chromosome abnormalities and euchromatic variants. J Med Genet 42: 609-629.

13. Cozaru GC, Butnariu LI, Gorduza EV (2012) Genetic Counseling in reproductive disorders. Procedia - Soc Behav Sci 33: 213-217.

14. Horsley SW, Knight SJ, Nixon J, Huson S, Fitchett M, et al. (1998) Del (18p) shown to be a cryptic translocation using a multiprobe FISH assay for subtelomeric chromosome rearrangements. J Med Genet 35: 722-726.

15. Maranda B, Lemieux N, Lemyre E (2006) Familial deletion 18p syndrome: case report. BMC Med Genet 7: 60. 